\title{
Теоретическое исследование фононного спектра и теплопроводности решетки в GeTe
}

\author{
(C) Д.А. Пшенай-Северин, А.А. Шабалдин, П.П. Константинов, А.Т. Бурков
}

Физико-технический институт им. А.Ф. Иофрфе Российской академии наук, 194021 Санкт-Петербург, Россия

E-mail: d.pshenay@mail.ru

Поступила в Редакцию 12 августа 2021 г.

В окончательной редакции 28 августа 2021 г.

Принята к публикации 28 августа 2021 г.

В последние годы возобновился интерес к термоэлектрическим материалам на основе теллурида германия, демонстрирующим высокую эффективность в среднетемпературном диапазоне. В данной работе обсуждаются особенности теоретического описания фононного спектра и решеточной теплопроводности в $\mathrm{GeTe}$ с использованием первопринципных методов. C их помощью рассчитана температурная зависимость решеточной теплопроводности в ромбоэдрической фазе и оценено ее изменение, связанное с рассеянием на точечных дефектах и при наноструктурировании. Исследована модификация фононного спектра при переходе к высокотемпературной кубической фазе. Проводится сравнение рассчитанных температурных зависимостей решеточной теплопроводности с имеющимися экспериментальными данными в GeTe и в твердых растворах на его основе.

Ключевые слова: теллурид германия, первопринципные расчеты, фононный спектр, теплопроводность решетки, наноструктурирование.

DOI: $10.21883 /$ FTP.2021.12.51697.12

\section{1. Введение}

Теллурид германия является перспективным среднетемпературным термоэлектрическим материалом. Сам GeTe и его твердые растворы с РbTе имеют высокую термоэлектрическую эффективность [1-5]. При этом содержание свинца в них невелико, что привлекательно с точки зрения экологических требований. В чистом GeTe присутствует большая концентрация вакансий германия, обусловливающая высокую концентрацию дырок [6]. Для оптимизации концентрации дырок в GeTe и в твердых растворах с РbТе использовалось легирование висмутом и сурьмой [1-5]. В твердых растворах $\mathrm{Ge}_{0.95} \mathrm{~Pb}_{0.05} \mathrm{Te}$, легированных висмутом, ZT достигает значений 1.5 , а в нелегированных растворах этого состава - до $1.1[1,2]$. Недавно были опубликованы результаты исследований твердых растворов $\mathrm{Ge}_{0.9} \mathrm{~Pb}_{0.1} \mathrm{Te}$, легированных сурьмой и висмутом, в которых были получены значения параметра $Z T$, значительно превышающие $2[3,4]$. Образцы без свинца с добавками индия и сурьмы демонстрировали $Z T=2.3[5]$.

Материалы с такой высокой термоэлектрической эффективностью, несомненно, привлекательны для использования в термоэлектрических генераторах. Однако эти экспериментальные результаты требуют независимого экспериментального подтверждения. Важно также понять, какие особенности электронной структуры и фононных спектров определяют высокую термоэлектрическую эффективность материалов на основе GeTe, что поможет выбрать пути ее дальнейшего повышения.

В последние годы интенсивно развиваются первопринципные методы расчета электронных и фононных спектров и транспорта. В данной работе приводятся результаты расчетов решеточных свойств GeTe в низкотемпературной ромбоэдрической $\alpha$-фазе, а также рассматривается модификация фононного спектра в высокотемпературной кубической $\beta$-фазе. Полученные результаты сравниваются с имеющимися в литературе, анализируется адекватность описания экспериментальных температурных зависимостей теплопроводности и возможные причины расхождений теоретических и экспериментальных результатов.

\section{2. Фононный спектр и решеточная теплопроводность в ромбоэдрической фазе GeTe}

Первопринципные расчеты электронной зонной структуры и фононного спектра в низкотемпературной ромбоэдрической фазе GeTe были проведены в довольно большом количестве работ. Исследовались структурные и электронные свойства в нормальных условиях [7] и под давлением [8]. Были рассчитаны упругие, диэлектрические свойства, фононный спектр GeTe, изучалось влияние возникновения мягких фононных мод на фазовый переход из низкотемпературной ромбоэдрической $\alpha$-фазы в высокотемпературную кубическую фазу, были выполнены расчеты теплопроводности решетки в $\alpha$-фазе [9-12].

В данной работе расчеты проводились в программе VASP $[13,14]$ в скалярно-релятивистском приближении, использовалась граничная энергия 250 эВ и сетка Монхорста-Пака $12 \times 12 \times 12$. Теоретические рав- 


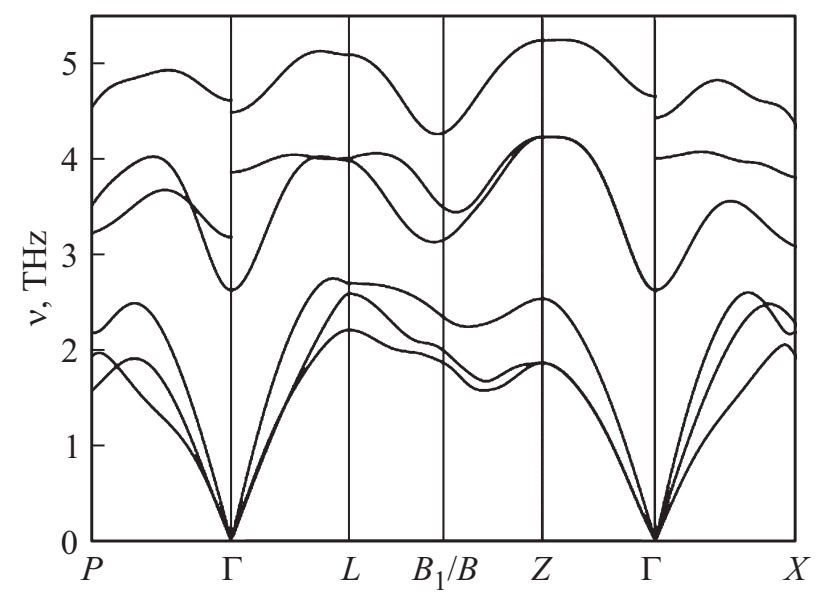

Рис. 1. Фононный спектр GeTe в ромбоэдрической фазе.

новесные значения параметров решетки $\alpha$-GeTe (пространственная группа $R 3 m$, № 160) оказались равны $a=4.3717 \AA$ и $\alpha=57.89^{\circ}$. Атом Ge располагался в начале координат, а Те - в позиции $(x, x, x), x=0.47$ в кристаллических координатах. Кроме того, с использованием теории возмущения для функционала плотности были рассчитаны борновские заряды (3.4 и 5.4 вдоль и поперек тригональной оси соответственно) и высокочастотная диэлектрическая проницаемость (35.1 и 39.5).

Фононный спектр и решеточная теплопроводность были рассчитаны в программе Phono3Рy [16,17] с использованием метода конечных смещений в суперъячейке. Силовые постоянные 2-го и 3-го порядков были рассчитаны в ромбоэдрической суперьячейке $4 \times 4 \times 4$ с учетом радиуса взаимодействия до $6.4 \AA$ для силовых постоянных 3-го порядка. Решеточная теплопроводность была рассчитана с учетом трехфононных процессов рассеяния в приближении времени релаксации, при этом интегрирование по зоне Бриллюэна проводилось на сетке $27 \times 27 \times 27$. Полученный фононный спектр приведен на рис. 1. Приведенные выше параметры решетки и спектр хорошо согласуются с полученными в работах $[11,12,18]$.

Результаты расчетов теплопроводности решетки приведены на рис. 2 в сравнении с экспериментальными данными из $[1,3,15]$. Для выделения экспериментального значения решеточной теплопроводности из полной использовался закон Видемана-Франца с числом Лоренца, определенным с учетом экспериментальных значений термоэдс по формуле, предложенной в [19]. Использование данных по термоэдс при вычислении числа Лоренца позволяет учесть степень вырождения носителей тока. В чистом GeTe расчет дает $\kappa_{p h}=3.2 \mathrm{BT} / \mathrm{MK}$ при комнатной температуре (кривая $l^{\prime}$ на рис. 2), тогда как экспериментальное значение - 2.5 Вт/мК. Наличие в образце атомов замещения и вакансий приводит к возникновению дополнительного механизма рассеяния на точечных дефектах, интенсивность которого зависит от атомной доли замещающих атомов $f_{i}$, разности масс замещающих атомов по сравнению со средней массой атомов $\left(\Delta M_{i} / \bar{M}\right)^{2}$ и от степени деформации решетки вокруг атома замещения [20]. Приблизительно оценить сверху величину теплопроводности решетки в присутствии точечных дефектов можно с учетом влияния только разности масс, тогда интенсивность рассеяния пропорциональна множителю $f_{M}=\sum_{i} f_{i}\left(\Delta M_{i} / \bar{M}\right)^{2}$. Если учесть таким образом рассеяние на вакансиях $\mathrm{Ge}$ c концентрацией 1.5 ат\%, соответствующей концентрации дырок $5 \cdot 10^{20} \mathrm{~cm}^{-3}$, то расчеты лучше согласуются с экспериментом (кривая $1^{\prime \prime}$ на рис. 2). Подобные расчеты, проведенные в [12], дали значения теплопроводности решетки в диапазоне 2.6-3.2 Вт/мК для чистого GeTe в зависимости от типа использованного приближения для функционала плотности, а в присутствии 3 ат\% вакансий $\mathrm{Ge}$ предсказывают снижение $\kappa_{p h}$ более чем в 2 раза при комнатной температуре. Для сравнения, в наших расчетах при такой концентрации вакансий получается снижение $\kappa_{p h}$ в 1.6 раза. На рис. 2 приведены результаты аналогичных расчетов для твердых растворов $\mathrm{Ge}_{0.96} \mathrm{Bi}_{0.04} \mathrm{Te}\left(2,2^{\prime}\right)$ и $\mathrm{Ge}_{0.91} \mathrm{Bi}_{0.04} \mathrm{~Pb}_{0.05} \mathrm{Te}$ (кривые $3,3^{\prime}$ ), для которых наблюдается неплохое согласие с экспериментальными данными из работы [1]. Видно заметное снижение теплопроводности, что вместе с оптимизацией концентрации дырок благоприятно сказывается на величине термоэлектрической добротности. На вставке рис. 2 приведены также зависимости $\kappa_{p h}$ от $f_{M}$ при $330 \mathrm{~K}$ в сравнении с экспериментальными значениями для нескольких образцов из $[1,3,15]$. В целом расчетная кривая неплохо описывает снижение теплопроводности

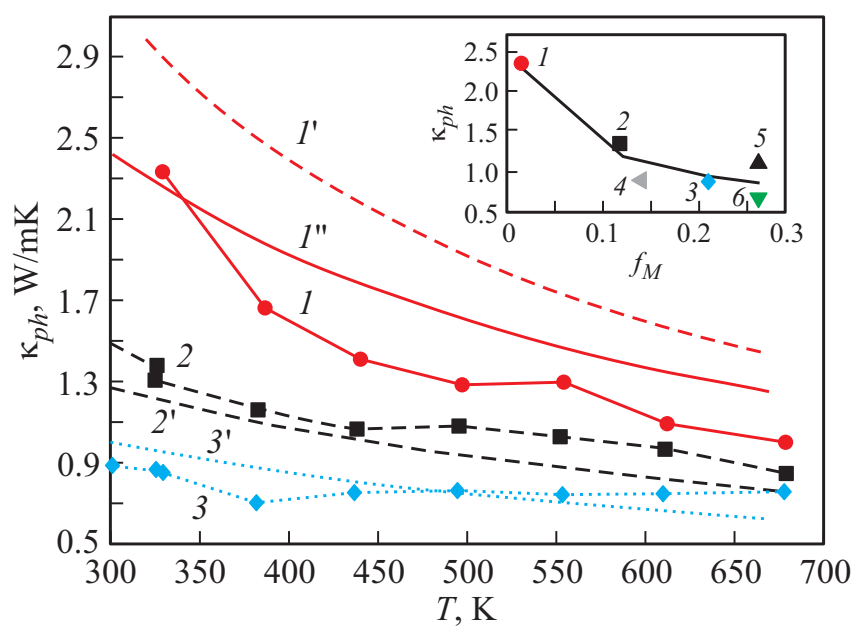

Рис. 2. Температурные зависимости решеточной теплопроводности $\kappa_{\mathrm{ph}}$. На вставке приведены зависимости $\kappa_{\mathrm{ph}}$ от фактора рассеяния за счет разности масс $f_{M}$ при $330 \mathrm{~K}$. 1-4 - экспериментальные данные из [1] для $\mathrm{GeTe}(1)$, $\mathrm{Ge}_{0.96} \mathrm{Bi}_{0.04} \mathrm{Te}(2), \mathrm{Ge}_{0.91} \mathrm{Bi}_{0.04} \mathrm{~Pb}_{0.05} \mathrm{Te}$ (3), $\mathrm{Ge}_{0.95} \mathrm{~Pb}_{0.05} \mathrm{Te}$ (4); $5-\mathrm{Ge}_{0.86} \mathrm{Bi}_{0.04} \mathrm{~Pb}_{0.1} \mathrm{Te}[15] ; 6-\mathrm{Ge}_{0.86} \mathrm{Bi}_{0.04} \mathrm{~Pb}_{0.1} \mathrm{Te}$ [3]. $I^{\prime}$ и $1^{\prime \prime}$ - расчет для GeTe без учета и с учетом присутствия вакансий $\mathrm{Ge} ; 2^{\prime}$ и $3^{\prime}-$ расчеты для соответствующих твердых растворов. 
решетки при увеличении концентрации примесей и усилении рассеяния на точечных дефектах, но видно, что для образцов 4-6 согласие с расчетом несколько хуже. Это может быть связано как с некоторым разбросом экспериментальных данных, так и с тем, что в расчетах не учитывались эффекты деформации решетки вокруг дефекта.

Используя полученные данные по времени релаксации фононов, можно оценить влияние наноструктурирования на $\kappa_{p h}$. Для этого удобно использовать величину кумулятивной теплопроводности решетки $\kappa_{\text {cum, }}$, показывающую долю вклада фононов с длиной свободного пробега меньше $l_{p h}$ в теплопроводность. Эта величина представлена на рис. 3. Рассеяние на границах зерен с размером $L$ должно снижать вклад в теплопроводность от фононов с $l_{p h}>L$. Например, при комнатной температуре для зерен с размером 20 нм можно ожидать снижения теплопроводности в $\mathrm{GeTe}$ на $20 \%$ и до $50 \%$ в твердых растворах на его основе. Это согласуется с выводами работы [21] о том, что рассеяние на точечных дефектах и на границах наиболее эффективны в различных диапазонах длин волн фононов и дополняют друг друга, приводя к большему снижению теплопроводности в твердых растворах по сравнению с чистым кристаллом. Аналогичный расчет для $600 \mathrm{~K}$ дал более слабое снижение (до 10-20\%) из-за уменьшения длины свободного пробега с температурой. Хотя в образцах $\mathrm{GeTe}$ электронный вклад в теплопроводность почти в 2 раза превышает решеточный, в твердых растворах эти вклады становятся сравнимыми и можно ожидать увеличения $Z T$ за счет граничного рассеяния. Например, в образце $\mathrm{Ge}_{0.91} \mathrm{Bi}_{0.04} \mathrm{~Pb}_{0.05} \mathrm{Te} \kappa_{h}=1.1 \mathrm{BT} / \mathrm{мK}$, а $\kappa_{p h}=0.86 \mathrm{BT} /$ мК. При неизменном дырочном вкладе и снижении решеточной части на $50 \%$ полная теплопроводность должна уменьшиться на $20 \%$. Если термоэдс и электропроводность не изменятся, то это должно дать такое же увеличение $Z T$.

\section{3. Фононный спектр в высокотемпературной кубической фазе GeTe}

При температуре 638-703 K в GeTe происходит фазовый переход из ромбоэдрической структуры в кубическую [22]. Схема расчета фононного спектра, использованная для $\alpha$-GeTe выше, в данном случае неприменима. Дело в том, что расчеты по методу функционала плотности, дающие силы межатомного взаимодействия, соответствуют нулевой температуре, но кубическая модификация GeTe при нулевой температуре является нестабильной. Поэтому атомные смещения, которые соответствуют ромбоэдрическому искажению кубической структуры, приводят к понижению энергии. Это приводит к возникновению отрицательных значений квадратов фононных частот для соответствующих мод (или мнимым частотам, которые на фононном спектре

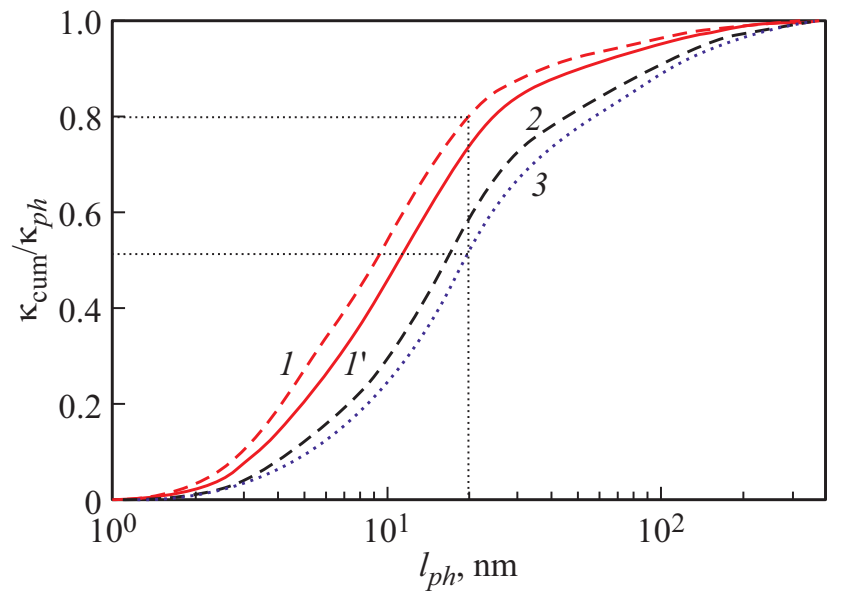

Рис. 3. Зависимость кумулятивной решеточной теплопроводности от длины свободного пробега фононов при комнатной температуре для GeTe без учета $(1)$ и с учетом $\left(1^{\prime}\right)$ вакансий $\mathrm{Ge}$, для $\mathrm{Ge}_{0.96} \mathrm{Bi}_{0.04} \mathrm{Te}(2), \mathrm{Ge}_{0.91} \mathrm{Bi}_{0.04} \mathrm{~Pb}_{0.05} \mathrm{Te}$ (3).

часто по соглашению изображаются отрицательными числами). Влияние мягких фононных мод на фазовый переход подробно обсуждалось в работе [11]. Для иллюстрации приведены результаты расчета фононного спектра для $\beta$-GeTe при теоретическом равновесном параметре решетки $a=6.014 \AA$ (рис. 4, слева). Видно, что имеются мнимые фононные моды в $\Gamma$ - и $X$-точках. Наибольшие по модулю мнимые моды в Г-точке соответствует оптическим колебаниям, в которых атомы $\mathrm{Ge}$ и Те смещаются в противоположных направлениях, что соответствует ромбоэдрическому искажению решетки.

Для корректного расчета фононного спектра в кубической фазе необходим учет ангармонических поправок к спектру. Такой расчет оказывается существенно более ресурсоемким. В данной работе был проведен расчет с использованием эффективного потенциала, зависящего от температуры (ТЭП) [23,24]. В этом методе потенциальная энергия ищется в виде ряда по атомным смещениям, но коэффициенты разложения уже зависят от температуры. Для их определения проводится моделирование движения атомов в кристалле по методу молекулярной динамики при заданной температуре. На каждом шаге моделирования сохраняются значения атомных смещений и сил межатомного взаимодействия. Эффективные силовые постоянные 2-го и более высоких порядков определяются с помощью метода наименьших квадратов по данным молекулярно-динамического расчета. Таким образом, получается эффективное гармоническое приближение (или приближение более высоких порядков) для описания потенциальной энергии при данной температуре. Полученные эффективные силовые постоянные позволяют использовать методы решеточной динамики для построения фононного спектра и расчета теплопроводности решетки.

Молекулярно-динамический расчет проводился с помощью программы VASP в NVT-ансамбле. В работе [6] 

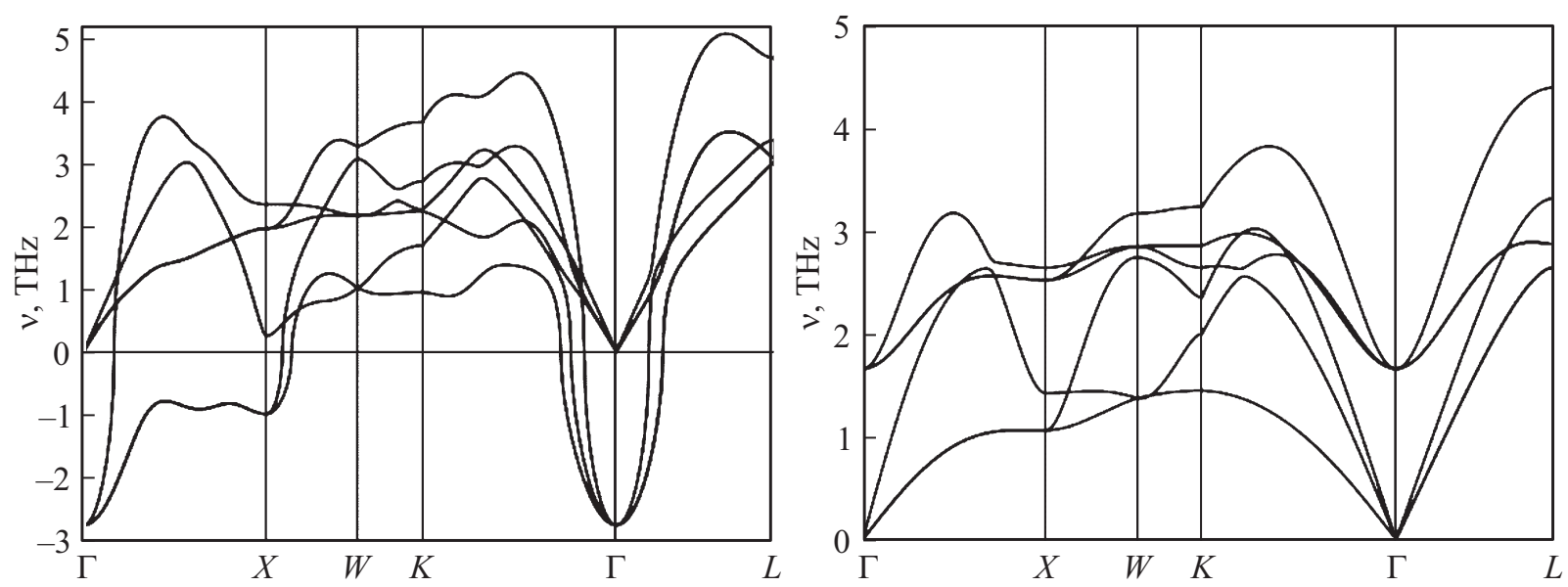

Рис. 4. Фононный спектр, рассчитанный в кубической $\beta$-фазе GeTe при $0 \mathrm{~K}$ (слева) и при $750 \mathrm{~K}$ (справа).

приводятся данные по рентгеновской дифракции GeTe, которые позволили определить температуру фазового перехода и параметры решетки. Переход в кубическую фазу происходил при температуре $>640 \mathrm{~K}$, а постоянная решетки увеличивалась и при $750 \mathrm{~K}$ (максимальной исследованной температуре) составила $a=6.022 \AA[6]$. Это значение постоянной решетки было использовано в расчете, который также проводился при $750 \mathrm{~K}$. Расчет проводился для суперьячейки $4 \times 4 \times 4$ (128 атомов), энергия обрезания была равна 250 эВ, а сетка Монхорста-Пака в зоне Бриллюэна суперъячейки была равна $2 \times 2 \times 2$. Шаг интегрирования уравнений движения был равен 1 фс, всего было промоделировано 7000 шагов. С использованием этих данных с помощью программы TDEP [23,24] были вычислены эффективные силовые постоянные 2-го и 3-го порядков для данной температуры.

Полученный в результате фононный спектр в кубической фазе GeTe приведен на рис. 4, справа, на котором видна стабилизация мнимых фононных мод при высокой температуре. Спектр согласуется с приведенным в работе [25], в которой был использован альтернативный подход и расчеты проводились при $800 \mathrm{~K}$ при равновесном для нулевой температуры теоретическом значении постоянной решетки $6.014 \AA$. В работе [25] силовые постоянные вычислялись с помощью итерационного алгоритма. Алгоритм стартовал со значений силовых констант при $0 \mathrm{~K}$. С помощью начальных значений силовых постоянных генерировался набор атомных смещений для данной температуры на основе эффективного гармонического приближения и вычислялись соответствующие межатомные силы. С использованием этих данных проводился поиск новых эффективных силовых постоянных до тех пор, пока не достигалась сходимость.

С использованием полученных силовых постоянных 2-го и 3-го порядков в программе TDEP была вычислена теплопроводность решетки в кубической фазе GeTe. Сравнение с экспериментальным значением теплопроводности решетки в $\mathrm{GeTe}$, которое при $800 \mathrm{~K}$ составляет $\sim 1.36-1.44$ Вт/мК [25], показывает, что полученное расчетное значение $(\sim 4 \mathrm{BT} / \mathrm{MK})$ оказывается в 2.6 раза выше экспериментального. В работе [25] было получено схожее расчетное значение $3.8 \mathrm{BT} / \mathrm{MK}$. Как было показано в работе [25], расхождение с экспериментом связано с тем, что в расчете учитывались только трехфононные процессы рассеяния. Учет четырехфононных процессов приводил к снижению расчетной теплопроводности на $\sim 55 \%$ в хорошем согласии с экспериментом. В то же время рассеяние на вакансиях Ge при высоких температурах не так эффективно. Оно дало снижение теплопроводности с 1.7 до $1.5 \mathrm{BT} / \mathrm{MK}$ при концентрации вакансий $2.1 \mathrm{aT} \% \quad\left(p_{h}=7.8 \cdot 10^{20} \mathrm{~cm}^{-3}\right)$, улучшая согласие с экспериментом [25]. В настоящей работе из-за ресурсоемкости данного расчета четырехфононные процессы рассеяния учтены не были.

\section{4. Заключение}

Таким образом, в данной работе на примере литературных данных и собственных расчетов проиллюстрировано, в какой мере современные первопринципные методы описания фононного спектра и решеточной теплопроводности применимы к такому довольно сложному объекту, как теллурид германия. Сложность его теоретического описания связана с изменением кристаллической структуры и фононного спектра при фазовом переходе из ромбоэдрической фазы в кубическую и с высокой концентрацией дырок из-за присутствия вакансий Ge.

Результаты расчетов решеточной теплопроводности $\alpha$-GeTе дали неплохое согласие с экспериментом при учете рассеяния на вакансиях германия и позволили оценить снижение теплопроводности в присутствии рассеяния на точечных дефектах замещения $(\mathrm{Pb}$ и $\mathrm{Bi})$. Согласие расчетов с экспериментом для твердых растворов с замещением $\mathrm{Ge}$ на $\mathrm{Pb}$ или $\mathrm{Bi}$ можно улучшить, более точно учитывая влияние модификации фононного 
спектра и времени жизни фононов в твердых растворах. Подобные методы расчета в настоящее время интенсивно развиваются (см., например, обзор [26]). Оценки влияния рассеяния на границах зерен на $\kappa_{p h}$ показали, что при размерах зерен 20 нм можно ожидать снижения теплопроводности решетки до $20 \%$ в чистом GeTe и до 50\% в твердых растворах при комнатной температуре. При более высоких температурах снижение теплопроводности будет ниже.

Описание свойств высокотемпературной $\beta$-фазы GeTe наиболее сложно, поскольку обычные расчеты по методу функционала плотности соответствуют нулевой температуре и не дают верного описания фононного спектра. Поскольку прямые методы неравновесной первопринципной молекулярной динамики слишком ресурсоемки, был использован метод эффективного потенциала, зависящего от температуры. Стабилизация мягких фононных мод за счет эффективного учета ангармонизма позволяет получить фононный спектр и рассчитать теплопроводность методами решеточной динамики. При этом для правильного описания теплопроводности решетки оказывается необходимым учесть четырехфононные процессы рассеяния.

\section{Финансирование работы}

Работа выполнена при поддержке Российского фонда фундаментальных исследований, грант № 18-52-80005 (БРИКС).

\section{Конфликт интересов}

Авторы заявляют, что у них нет конфликта интересов.

\section{Список литературы}

[1] Л.В. Прокофьева, Ю.И. Равич, Д.А. Пшенай-Северин, П.П. Константинов, А.А. Шабалдин. ФТП, 46, 889 (2012).

[2] T. Parashchuk, A. Shabaldin, O. Cherniushok, P. Konstantinov, I. Horichok, A. Burkov, Z. Dashevsky. Physica B: Condens. Matter, 596, 412397 (2020).

[3] J. Li, X. Zhang, Z. Chen, S. Lin, W. Li, J. Shen, I.T. Witting, A. Faghaninia, Y. Chen, A. Jain, L. Chen, G.J. Snyder, Y. Pei. Joule, 2, 976 (2018).

[4] J. Li, X. Zhang, X. Wang, Z. Bu, L. Zheng, B. Zhou, F. Xiong, Y. Chen, Y. Pei. J. Am. Chem. Soc., 140, 16190 (2018).

[5] M. Hong, Z.-G. Chen, L. Yang, Y.-C. Zou, M.S. Dargusch, H. Wang, J. Zou. Adv. Mater., 30, 1705942 (2018).

[6] E.M. Levin, M.F. Besser, R. Hanus. J. Appl. Phys., 114, 83713 (2013).

[7] K.M. Rabe, J.D. Joannopoulos. Phys. Rev. B, 36, 3319 (1987).

[8] A. Ciucivara, B.R. Sahu, L. Kleinman. Phys. Rev. B, 73, 214105 (2006).

[9] R. Shaltaf, X. Gonze, M. Cardona, R.K. Kremer, G. Siegle. Phys. Rev. B, 79, 075204 (2009).

[10] R. Shaltaf, E. Durgun, J.-Y. Raty, Ph. Ghosez, X. Gonze. Phys. Rev. B, 78, 205203 (2008).

[11] U.D. Wdowik, K. Parlinski, S. Rols, T. Chatterji. Phys. Rev. B, 89, 224306 (2014)
[12] D. Campi, L. Paulatto, G. Fugallo, F. Mauri, M. Bernasconi. Phys. Rev. B, 95, 024311 (2017).

[13] G. Kresse, D. Joubert. Phys. Rev. B, 59, 1758 (1999).

[14] G. Kresse, J. Furthmüller. Phys. Rev. B, 54, 11169 (1996).

[15] A.A. Shabaldin, P.P. Konstantinov, A.Y. Samunin. XVII Int. Conf. Thermoelectrics and Their Applications (ISCTA 2021), St. Petersburg, Russia, September 13-16, 2021.

[16] A. Togo, L. Chaput, I. Tanaka. Physical Review B 91, 094306 (2015).

[17] K. Mizokami, A. Togo, I. Tanaka. Phys. Rev. B, 97, 224306 (2018).

[18] V. Askarpour, J. Maassen. Phys. Rev. B, 100, 075201 (2019).

[19] H.-S. Kim, Z.M. Gibbs, Y. Tang, H. Wang, G.J. Snyder. APL Materials, 3, 41506 (2015).

[20] P.G. Klemens. Proceedings of the Physical Society. Section A, 68, 1113 (1955).

[21] H.J. Goldsmid, H.B. Lyon, E.H. Volckmann.Proc. 14th Int. Conf. on Thermoelectrics (St. Petersburg, Russia, 1995) p. 16.

[22] Р.С. Ерофеев. Изв. АН СССР, Неорг. матер., 14, 1422 (1978).

[23] O. Hellman, I.A. Abrikosov. Phys. Rev. B, 88, 144301 (2013).

[24] O. Hellman, I.A. Abrikosov, S.I. Simak. Phys. Rev. B, 84, 180301(R) (2011).

[25] Y. Xia, M.K.Y. Chan. Appl. Phys. Lett., 113, 193902 (2018).

[26] L. Lindsay, A. Katre, A. Cepellotti, N. Mingo. J. Appl. Phys., 126, 050902 (2019)

\section{Theoretical investigation of the phonon spectrum and the lattice thermal conductivity in GeTe}

\author{
D.A. Pshenay-Severin, A.A. Shabaldin, \\ P.P. Konstantinov, A.T. Burkov \\ loffe Institute, \\ 194021 St. Petersburg, Russia
}

\begin{abstract}
Recently, there has been renewed interest in thermoelectric materials based on germanium telluride, which demonstrate high efficiency in mid-temperature range. This paper discusses the theoretical description of the phonon spectrum and lattice thermal conductivity in GeTe using ab initio methods. Using these methods, the temperature dependence of the lattice thermal conductivity in the rhombohedral phase was calculated and effects of scattering by point defects and nanostructuring were estimated. The modification of the phonon spectrum upon the transition to the high-temperature cubic phase is investigated. The calculated temperature dependences of the lattice thermal conductivity are compared with the available experimental data on GeTe and its solid solutions.
\end{abstract}

solid solutions. 